\title{
Constrained evolutionary optimization of a distillation train in Chemical Engineering
}

\author{
Roberto Gutiérrez-Guerra \\ Department of Chemical Engineering \\ Rodolfo Murrieta-Dueñas \\ Department of Chemical Engineering Department of Chemical Engineering \\ Universidad de Guanajuato, Gto., Mexico Universidad de Guanajuato, Gto., Mexico Universidad de Guanajuato, Gto., Mexico \\ Email: uranio_bz@hotmail.com \\ Email: rodol_99@yahoo.com.mx \\ Email: iq_jcg@hotmail.com
}

\section{Arturo Hernández-Aguirre \\ Computer Science Department}

Center for research in Mathematics, CIMAT, Gto., Mexico

Email: artha@cimat.mx

\author{
J.G. Segovia-Hernández \\ Department of Chemical Engineering \\ Universidad de Guanajuato, Gto., Mexico \\ Email: gsegovia@ugto.mx
}

\begin{abstract}
The optimal design and synthesis of distillation systems remains one of the most challenging problems in process engineering. The goal of this paper is to introduce an evolutionary approach for the optimization of the total energy consumption of distillation systems with constraints. Moreover, the contribution of this paper is a novel constraint handling technique that manages design goals as equality constraints, such as the purity and the recovery of the final components. In the literature of these problems prevail the use of inequality constraints; although easy to apply they may lead the search to suboptimal solutions. The case study is a distillation column sequence (DCS) for the separation of four components; this problem is easy to describe yet complex to solve so our approach can show its advantages. The evolutionary algorithm Boltzmann Univariate Marginal Distribution Algorithm, (BUMDA), performs the optimization. AspenONEC)software is used for the rigorous evaluation of the fitness function of the population. The results show the efficacy performance of the proposed approach reaching near optimal designs in less than 3000 function evaluations.
\end{abstract}

Keywords-Optimization of distillation sequences, EDAs, Boltzmann Distribution

\section{INTRODUCTION}

The distillation process is one of the most studied and utilized separation methods in the chemical industry. It is well known that such a process demands high levels of energy consumption for its normal operation, but also it is known that they are highly inefficient. A large amount of research work has been done to improve the energy efficiency of distillation systems, focusing in either the design of optimal distillation schemes, or improving internal column efficiency. For the sake of a visual and improved introduction to the distillation problem, the typical distillation column is shown in Figure 1. The column has $N_{T}$ stages, there is a feed location at some stage $N_{F}, 1 \leq N_{F} \leq N_{T}$. In that location a mixture is feed into the column. End product $C 1$ is obtained at the top of the column after passing through a Condenser $(C o n d)$. From the condenser some portion of $C 1$ is returned into the tower in an amount called Reflux Ratio $(R R)$. From the bottom of the tower the rest of the mixture (without $\mathrm{C} 1$ ) passes through a reboiler (Reb1) and some portion is taken back into the tower. The design variables are $N_{T}, N_{F}$, and $R R$; the optimization

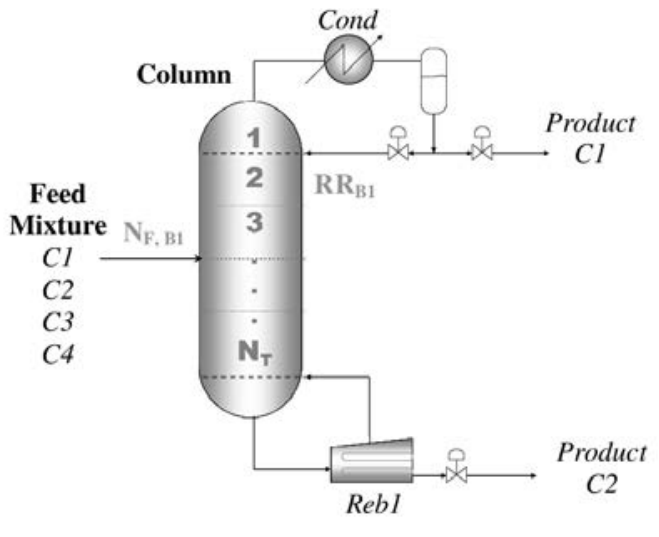

Fig. 1. Distillation column to split two components

goal is to find the values for such variables that minimize the total heat duty $(\mathrm{Q})$, of the column, subject to a desired value of purity and recovery of each product. An additional (inequality) constraint is the maximum number of stages of the column. In this paper, all computations of the column model are performed by the commercial software AspenONE (C). The purity and the recovery are values that the design tool has to achieve with high precision, otherwise, the column is useless if the purity or recovery are off the target. Likewise, the column becomes more expensive if the products are obtained with a purity greater than the requested target.

The optimization of any distillation system is a computer problem of large size with a significant number of strongly nonlinear equations that causes serious numerical difficulties. For many years the deterministic approaches (i.e. gradient based) have been developed and applied to the design of DCS, however, the evolutionary based approaches have only been explored with some success in the recent years.

Deterministic strategies tackle the problem considering the distillation sequence as superstructures which, basically, have been solved with mixed-integer linear programming and nonlinear programming approaches. Other approaches consider reduced models ([1], [2], [3], [4], [5], [6], [7], [8], [9], [10]). Some of these reduced methods can provide useful results, 
however, it would be desirable to incorporate rigorous models in the synthesis procedures in order to increase their industrial relevance and scope of application, particularly for non-ideal mixtures. These rigorous MINLP synthesis models exhibit significant difficulties, such as: equations that can become singular, the solution of many redundant equations, and the requirement of good initialization points. Computing a solution with any of these methods is a very intensive number crunching task.

The stochastic algorithms can deal with multi-modal and non-convex problems in a effective way, and reducing the mentioned limitations. They are a suitable alternative for the design and optimization of complex separation schemes using rigorous models [11]. Evolutionary algorithms have been applied to chemical engineering design with some success ([12], [13], [14], [15], [16]). In this paper an Estimation of Distribution Algorithm (EDA) called Boltzmann Univariate Marginal Distribution Algorithm (BUMDA) [26] is used for the optimization of distillation column sequences (DCS). A constraint handling technique that deals with equality and inequality constraints is also proposed.

The structure of this paper is as follows: in Section II an introduction to distillation columns and to the distillation problem is presented. Section III presents a review of evolutionary approaches applied to the design of DCS. Section IV describes the parameters of a distillation column, whereas Section V presents the Boltzmann Univariate Marginal Distribution Algorithm (BUMDA), and the constraint handling technique adopted for the design of DCS. Section VI is the experimental section and presents the case study: optimization of distillation column sequence to separate a mixture of 4 components. In Section VII the results of the experiments are discussed from several perspectives, and in Section VIII the conclusion of this work are provided.

\section{Distillation FUNDAMENTALS AND THE DISTILLATION PROBLEM}

Simply speaking, distillation is the process of vaporizing a liquid, condensing the vapor, and collecting the condensate in another container. This technique is useful for separating a liquid mixture when the components have different boiling points, or when one of the components will be distilled. It is one of the principal methods of purifying a liquid. Distillation can be defined as: a process in which a liquid or vapor mixture of two or more substances is separated into its component fractions of desired purity, by the application and removal of heat. Distillation is based on the fact that the vapor of a boiling mixture will be richer in the components that have lower boiling points. Therefore, when this vapor is cooled and condensed, the condensate will contain more of the volatile components. At the same time, the original mixture will contain more of the less volatile material. In the traditional distillation of a pure substance, vapor rises from the distillation flask (reboiler) and comes into contact with a thermometer that records its temperature, the vapor then passes through a condenser, which condenses the vapor and passes it into the receiving flask (condenser) [38]. See Figure 1.

The reflux ratio plays an important role in the column design: to small reflux corresponds large number of stages. Likewise, for large reflux the column is shorter (with less stages), therefore cheaper. In continuous distillation the reboiler and the condenser consume large amounts of energy. When the purity and recovery targets are introduced into this formulation we get a complex trade-off of variables for the optimization problem. For instance, a larger reflux ratio would increase the energy consumption and the tower diameter but it decreases the number of stages with undesirable effects on the purity of the product [38].

The distillation columns can be designed with the short or the rigorous method. The short methods are used to estimate the minimum number of stages and the minimum reflux ratio. The rigorous methods work with the MESH equations (Mass, Equilibrium, Sum and Heat), and the distillation column model is a cascade of stages (the ouput of one stage is the input to the next). Thus, the MESH equations are solved for each stage.

As mentioned, this set of equations provides the mathematical model for a distillation column. In any case, the model shows a coupled structure such that the full set of equations must be solved simultaneously. The complete design of the distillation system should provide the tray structure of each column and the operating conditions (such as pressure and temperature) that minimize a given objective function, for instance, the total yearly cost. The design of such distillation system yields a complicated optimization problem which requires a mixed-integer nonlinear programming formulation.

The MESH equations are a highly non linear problem specially difficult to solve due to the phases equilibria relationships. This means that it is necessary to include a model that describes the system behavior through the phases equilibrium relationship. There are different models, for example ChaoSeader, UNIQUAC and NRTL, which are applied in agreement with the mixture characteristics. In this paper the Chao-Seader model is chosen, and Aspen Plus instructed to use it for the model computation.

\section{EVOLUTIONARY DESIGN OF CONTINUOS Distillation COLUMNS: RELATED WORK}

Evolutionary algorithms have been applied to column design in chemical processes, however, the application is not as common as in other areas of engineering. The following description is an account of interesting contributions found in the specialized literature: Fraga and Matas [12], used genetic algorithms to optimize batch distillation columns to split three components azeotropic mixtures. Thereafter, Kolbe and Wenzel [27], established a genetic algorithm to design dividing wall distillation columns used in the petrochemical cuts. This algorithm reduces the difficulties of convergence and the number of rigorous simulations required of these columns; the fitness function in this algorithm is the minimization of total annual cost, and the results of the implementation of such algorithm showed important advantages in the design obtained for this distillation sequence. Later, Tarafder et al., [28], made an analysis about optimal possible solutions of chemical processes using multi objective optimization and genetic algorithms. Results discussed the Pareto front of several design problems. At the same time, Gutierrez-Antonio [29], presented a study of analysis, design and optimization of azeotropic distillation columns. The design methodology is based on genetic algorithm, where the goal is the minimization of both the heat 
duty and the stages number while considering as constraints the purity of the components. Results showed that this technique is able to find the minimum energy consumption design, reducing the possibility to get trapped in local minima. Later, Gomez et al.,[30] proposed a design and optimization methodology base on genetic algorithms coded on MatLab(c)and implementing rigorous simulations made in the processes simulator Aspen Plus(c). The methodology was applied to design Petlyuk columns. The results demonstrated important energy savings and increasing of thermodynamic efficiency and reduction of capital costs compared with the conventional sequence, besides, the configuration was able to get better control properties than the conventional sequence. Vázquez-Castillo et al., [31] achieved the intensified distillation systems optimization to separate four components mixtures, applying a multi objective genetic algorithm with constraint handling, linked to Aspen Plus(c)to evaluate the fitness function. Throughout their study, they determined the dependency of the relative volatility on the energetic consumption, the second law of the thermodynamic, the total annual cost and the control properties on intensified systems. The numeric performance showed that this design tool is robust and it can be used to design coupled distillation sequences to split multicomponent mixtures. At the same time, Gutiérrez-Antonio and Briones [32], implemented a multiobjective genetic algorithm with constraint handling to obtain the Pareto front of Petlyuk sequences. This algorithm was also coupled with Aspen Plus(C)to solve the complete model represented by the $M E S H$ equations. Although their approach uses a shortcut method the results show that design optimization based on shortcuts can be applied to either chemical or petrochemical plants. Bozorgmehry [39], presents an approach based on the prior modeling by short-cut methods of a permissible set of column architectures, and then applies a Genetic Algorithm to find the best combination of the allowed structures that separates an input mixture and minimizes the Total Annual Cost. Also, Bozorgmehry [40], introduces a novel procedure for the optimum synthesis of distillation column sequences. In the mentioned works of Bozorgmehry, the constraint handling is not an issue because the approach is based on short-cut models, therefore, calculating the equations is computational intensive and complex but the process stops on approximately desired specifications. Recenlty, VázquezOjeda et al.,[33], used the Differential Evolution algorithm to optimize distillation columns, having as fitness function the minimization of energy consumption (reducing the number of reboilers and condensers) in multicomponent systems. The results demonstrated important energy savings. None of the approaches reviewed before use equality constraints with the evolutionary method; they only deal with inequality constraints. Therefore, the desired purity of the components is incorporated as "more than $98.5 \%$ " for simplification purposes (and all of its undesirable consequences). This paper, however, introduces a constraint handling method that manages equality constraints, therefore, it is able to reach exact design targets, for instance, a purity of $98.5 \%$.

\section{DESIGN AND OPTIMIZATION OF A DISTILLATION} TRAIN

The objective of a distillation train is to separate a multicomponent mixture using only single columns. Each column has a feed stage and carries out the distillation of two adjacent

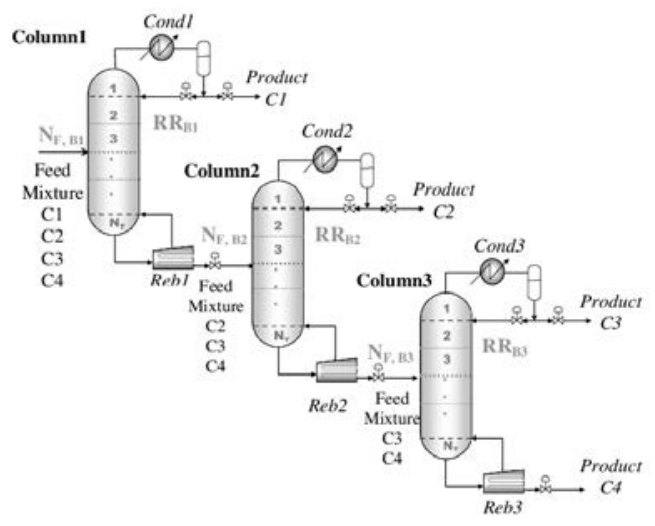

Fig. 2. Flowsheet of distillation train to split a four components mixture

components (adjacent volatility), and delivers two products: one at the top and one at the bottom. In order to separate a mixture of $N$ components it is necessary to use $N-1$ single columns [17]. In zeotropic mixtures the order in which the components are separated is based on their relative volatility. In this problem a mixture four components is fed $(C 1, C 2, C 3$ and $C 4)$. The first component $(C 1)$ delivered is the most volatil and it is obtained at the top (Cond1) of the first column; while the rest of the components are obtained at the bottom (Reb1) of the column. In the second column, $C 2$ is separated in the condenser 2 (Cond2); finally, the last column separates the last two products: one at the top $(C 3, C o n d 3)$ and one at the bottom $(C 4, R e b 3)$. The distillation train to purify this mixture is shown in Figure 2. The basic design of each distillation column requires: the maximum number of stages $\left(N_{T}\right)$, feed stage $\left(N_{F}\right)$ and reflux ratio $(R R)$ (which is the relationship between the liquid flow $L$ that returns to the column and the vapor flow $V$ that rises in the column). With this set of variables the main design goal is the minimization of the total heat duty subject to the specified purity and recovery of each product.

\section{OPTIMIZATION STRATEGY}

In this work we implement the Boltzmann Univariate Marginal Distribution Algorithm (BUMDA), a class of EDA which was designed after the Boltzmann distribution [26]. It is well known that this distribution is well defined for an infinite number of samples, thus an approximation is taken to overcome the problem of a limited size population. BUMDA assumes the population is infinite so the Boltzmann distribution is known everywhere. Then, using a sample of finite size, the parameters of the Normal distribution that best approximates the Boltzmann distribution in the sense of the KullbackLeibler divergence are computed. The mean and variance of the Normal distribution are computed as shown in Figure 3. The BUMDA algorithm is quite simple. A population is initialized between the bounds of the search space, and the fitness function calculated. $20 \%$ of the best individuals are stored in the selected set, and used to compute the mean and variance parameters of the Normal distribution. Then new individuals are simulated from a Normal distribution with such parameters to populate the next generation. BUMDA performs a re-start procedure of the population which is triggered when the variance is reset after reaching some threshold value. The 
initial threshold for the variance is 1.0 , however, this value is reduced to a new value which is a factor of 0.5 of the previous. The re-start step promotes diversity and exploration of the population as it can be observed in Figure 5. The algorithm Iterates for a predefined number of generations.

The BUMDA algorithm includes a constraint handling method for equality and non-equality constraints. An equality constraint is converted to an inequality with an $\epsilon$ threshold. For instance, the equality constraint:

$$
g(x)-k=0
$$

is treated as:

$$
|(g(x)-k)| \leq \epsilon
$$

Thus, the constraint is evaluated to true when $(g(x)-k)$ is inside the interval $[-\epsilon,+\epsilon]$. The threshold $\epsilon$ is reduced to the half when the whole population makes true all the constraints.

The optimization approach described in this paper has been developed using an interface which links the optimizer BUMDA (Master, coded in MatLab(c) with the modular simulator Aspen Plus(C)(Fitness Function Evaluator) through the Excel(C)(Date Base). In this interface the BUMDA algorithm needs one individual physically feasible to begin the optimization process. Here, physically feasible implies that for a distillation column, the feed stage must be smaller than the total number of stages, and the reflux ratio is greater than zero. The BUMDA algorithm generates individuals with the proper dimensionality, which are sent to Aspen Plus(c), one by one, in order to simulate the distillation scheme and get the values of the fitness function evaluated with constraints. With this information, BUMDA algorithm sorts the individuals according to the penalization strategy described in Figure 4, and the best individuals are chosen to compute the parameters of the Normal distribution that will populate the next generation. It is important to mention that during the simulation process, an infinite value (worst value) is assigned to the fitness function of the individuals whose simulation was impossible for Aspen Plus (Step 1 of constraint handling). Also, in that same condition, the purities are set to a "bad" value (0.01). If an individual is feasible its Aspen evaluation is returned at once. In Step 2 of constraint handling, the fitness function is penalized by a factor proportional to the amount the constraint is violated. In Step 3, the penalization factor is made larger and proportional to the number of constraints not valid. In Step 4, a number of stages larger than the maximum allowed increases the penalization factor applied the fitness function. Likewise, candidates with a small number of stages (and below the threshold), are favored in greater amount than the candidates with a larger number of stages (also below the threshold).

\section{STUDY CASE: OPTIMIZATION OF A DISTILLATION TRAIN}

We study a mixture made of four lineal aliphatic hydrocarbons fed at a flow-rate of $45.36 \mathrm{kmol} / \mathrm{h}$ in the first column as saturated liquid. The characteristics and the purity and recovery design goals (equality constraints) of the mixture are presented in Table I. The proportion of each component is shown in mol fraction. Each component must be delivered with the specified purity and recovery. The design pressure for the separation was chosen to ensure the use of cooling water in the condensers.

\section{Constraint Handling for Distillation Train Design}

1) Evaluate individual $I$

$Q_{\text {total }} \leftarrow$ AspenPlus $(I)$

If (convergence(Aspen) $==$ false $)\{$

$Q_{\text {fitness }}=1 E 9 ; x_{i}^{\text {pur }}=0.01 ; x_{i}^{\text {rec }}=0.01$;

$w_{i}^{\text {pur }}=0 ; w_{i}^{r e c}=0$;

viola $^{\text {pur }}=0$; viola $^{r e c}=0$;

Return $\left.\left(Q_{\text {fitness }}\right) ;\right\}$

2) Penalize $Q_{\text {total }}$ with the violated constraints of purity and recovery of each component

$Q^{\text {pur }}=0$;

For $\mathrm{i}=1$ to \#Components

If $\left(\right.$ abs $\left.\left(x_{o_{i}}^{\text {pur }}-x_{s_{i}}^{\text {pur }}\right)>\epsilon\right)\{$

$w_{i}^{\text {pur }}=10\left(x_{o_{i}}^{\text {pur }}-x_{s_{i}}^{\text {pur }}\right)^{2}$;

$Q^{\text {pur }}=Q_{\text {total }}+w_{i}^{\text {pur }} \times Q_{\text {total }}$;

viola $^{\text {pur }}=$ viola $\left.^{\text {pur }}+1 ;\right\}$

EndFor

$Q^{r e c}=0$

For $\mathrm{i}=1$ to \#Components

If $\left(\right.$ abs $\left.\left(x_{o_{i}}^{r e c}-x_{s_{i}}^{r e c}\right)>\epsilon\right)\{$

$w_{i}^{r e c}=10\left(x_{o_{i}}^{r e c}-x_{s_{i}}^{r e c}\right)^{2}$;

$Q^{r e c}=Q_{\text {total }}+w_{i}^{r e c} * Q_{\text {total }}$;

viola $^{\text {rec }}=$ viola $\left.^{\text {rec }}+1 ;\right\}$

$Q_{\text {pen }}=Q_{\text {total }}+Q^{\text {pur }}+Q^{\text {rec }}$

3) Penalize $Q_{\text {pen }}$ by the number of constraints violated

$$
\begin{aligned}
& \text { If }\left(\left(\text { viola }^{\text {pur }}+\text { viola }^{\text {rec }}\right)>0\right)\{ \\
& w_{\text {num_pen }} \leftarrow \text { GetFactors_w }\left(C_{\text {viola }}\right) \\
& \left.Q_{\text {num_pen }}=w_{\text {num_pen }} * Q_{\text {pen }} ;\right\} \\
& \text { else } \\
& Q_{\text {num_pen }}=Q_{\text {pen }} ;
\end{aligned}
$$

4) Penalize $Q_{\text {num_pen }}$ by \#Stages in each column and get $Q_{\text {fitness }}$

$N_{T \text { stages }}=N_{1}+\cdots+N_{k}$;

If $\left(N_{T_{-} \text {stages }}<N_{\text {max_stages }}\right)\{$

$w_{\text {stages }}=\frac{N_{T_{\text {_stages }}}}{N_{\text {mar_stages }}} ;$

$Q_{\text {pen_stages }}=\bar{w}_{\text {stages }} \times Q_{\text {num_pen }} ;$

$\left.Q_{\text {fitness }} \leftarrow Q_{\text {pen_stages }} ;\right\}$

else

$Q_{\text {fitness }} \leftarrow Q_{\text {pen_stages }}$;

5) Return $\left(Q_{\text {fitness }}\right)$

Fig. 4. The constraint handling technique used with BUMDA for the optimization of a distillation train

TABLE I. CHARACTERISTICS OF CASE OF STUDY 1

\begin{tabular}{c||c||c||c||c}
\hline \multicolumn{3}{c||}{ Characteristics of the mixture } & \multicolumn{2}{c}{ Design Constraints } \\
\hline ID & Component & Feed, mol fraction & Purity & Recovery \\
\hline \hline C1 & n-butane & 0.05 & 0.987 & 0.98 \\
C2 & n-pentane & 0.45 & 0.98 & 0.98 \\
C3 & n-hexane & 0.45 & 0.98 & 0.98 \\
C4 & n-heptane & 0.05 & 0.986 & 0.98 \\
\hline
\end{tabular}

The phase equilibrium for liquid of this mixture is calculated with the Chao-Seader model, because is usually recommended for hydrocarbon mixtures operating at low or medium pressure ([35]).

As mentioned, BUMDA performs the optimization of the total heat duty (fitness function, $Q$ ) subject to design constraints, with continuous and discrete variables. The variables 


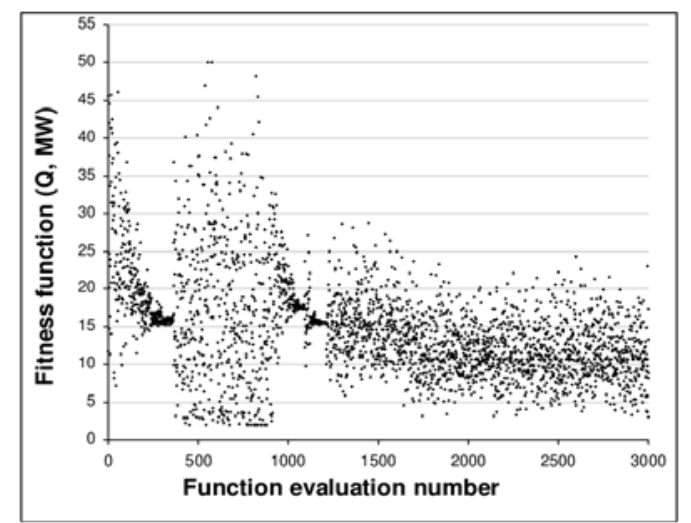

Fig. 5. Behaviour of the BUMDA algorithm with re-start

are: Total stages number, feed stage location, and reflux ratio for each column, shown in Equation 1. In overall, the optimization problem is integrated by 9 variables, out of which 6 are discrete and 3 continuous (reflux ratio of each column). The problem is stated as follows:

Minimize Total Heat Duty $Q_{T}$

$$
Q_{T}\left(N_{T, B 1}, N_{T, B 2}, N_{T, B 3}, N_{F, B 1}, N_{F, B 2}, N_{F, B 3}, R R_{B 1}, R R_{B 2}, R R_{B 3}\right)
$$

Subject to:

$$
\begin{aligned}
& x_{o_{i}}^{\overrightarrow{p u r}}=x_{s_{i}}^{\overrightarrow{p u r}} \\
& x_{o_{i}}^{\overrightarrow{r e c}}=x_{s_{i}}^{\overrightarrow{r e c}}
\end{aligned}
$$

Where: $N_{T, B 1}, N_{T, B 2}$ and $N_{T, B 3}$ are total stages number; $N_{F, B 1}, N_{F, B 2}$ and $N_{F, B 3}$ are the feed stage locations; and $R R_{B 1}, R R_{B 2}$ and $R R_{B 3}$ are reflux ratios, in each column; $x_{o_{i}}^{\overrightarrow{p u r}}, x_{o_{i}}^{\overrightarrow{r e c}}$ and $x_{s_{i}}^{\overrightarrow{p u r}}, x_{s_{i}}^{\text {rec }}$ are the vectors of required and obtained purities and recoveries, respectively; $\epsilon=3 E-4$.

For this study case 3000 fitness function evaluations were made with a population of 30 individuals. The time required to perform the optimization is about 6 hours. The simulation was run on a PC computer with i5 processor core, clock frequency at $2.8 \mathrm{GHz}$, and $16 \mathrm{~GB}$ of RAM. As mentioned, Figure 5 shows the behavior of the BUMDA algorithm with re-start on our minimization problem. It is important to note that each time this mechanism is activated the fitness function value is again minimized, therefore, the re-start procedure is useful.

Figure 6 shows the best results obtained from 30 runs. The minimum fitness function value is $7.30 \mathrm{MBTU} / \mathrm{h}$ and the worst is $14.30 \mathrm{MBTU} / \mathrm{h}$. The descriptive statistics of the fitness function are: average, median, standard deviation and variance, with values $10.05,9.52,1.96,3.87$, respectively.

\section{DISCUSSION OF THE RESULTS}

From the results obtained once the design constraints have been satisfied or at least they are considerably accepted, both the heat duty and the stage number are taken as the representative parameters which will give us an idea about the feasibility of the design keeping on mind economical, dynamic performance and environmental sceneries. Keeping this view in mind, and remembering the trade-off between stages number and heat duty (the greater the number of stages

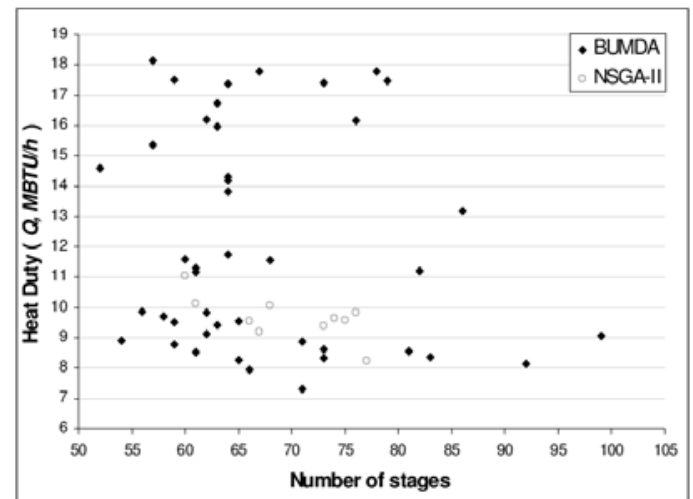

Fig. 6. Comparative behavior of the best individuals of BUMDA vs NSGA-II.

the less the heat duty), a very worthy analysis is derived from a detailed study of their behavior. Thus, when these are plotted as shown in the Figure 6 (as black dots), we can see that there exists a distribution which is found between 50 to 100 stages, and with a heat duty range located from 7.3 $\mathrm{MBTU} / \mathrm{h}$ to $15 \mathrm{MBTU} / \mathrm{H}$. At the same time, it is important to observe that most of the solutions lie on a range of 55 and 75 stages, and between 7.3 and $12 \mathrm{MBTU} / \mathrm{h}$. Only a few isolated points are located after stage 80 . Furthermore, it is essential to note that the best compromise (the combined effect of all variables), is located at stage 72 and $7.3 \mathrm{MBTU} / \mathrm{h}$. In fact, it can be established that the best design is precisely one whose minimum fitness function value has the the average number of stages. In terms of design this solution represents a good candidate for further analysis, such as dynamic performance, economical feasibility and environmental impact. Furthermore, in Figure 6 there are additional points which correspond to the best values obtained with experiments carried out using the NSGA-II multiobjective optimization algorithm. In this plot, and considering the same two variables in discussion (heat duty and stages number), it is possible to observe that the values generated by the BUMDA algorithm present a better behavior on both variables. We can easily state this because graphicaly one can see that the BUMDA solutions dominate the NSGA-II solutions. This mean that, in general terms, the BUMDA design will be economically cheaper and would cause less environmental impact (due to the shorter columns and the use of less energy the carbon dioxide emissions will be reduced). In conclusion, the best NSGA-II design obtained 77 stages and a heat duty equal to $8.3 \mathrm{MBTU} / \mathrm{h}$. These results were improved by our approach with a design with 72 stages and $7.3 \mathrm{MBTU} / \mathrm{h}$.

The best and worst solutions obtained with BUMDA Algorithm are presented in Table II. They are compared with the best results reported by [37] using the same distillation train and same characteristics of the mixture. As it can be observed, the best design found by the BUMDA algorithm needs less total stage number while in terms of energy it requires approximately the same total heat duty. In addition, considering the purity as one of the most important constraints, the BUMDA algorithm meets the target values within a very little tolerance. The other approach exceeds this value on all components (do not use equality constraints).

A more detailed explanations will allow us to identify more 
TABLE II. FINAL RESUlTS OF Distillation TraiN

\begin{tabular}{|c|c|c|c|}
\hline & \multicolumn{2}{|c|}{ BUMDA Algortihm } & NSGA-II \\
\hline Design & Worst & Best & Best \\
\hline Heat Duty $Q, M B T U / h$ & 14.30 & 7.30 & 8.23 \\
\hline \multicolumn{4}{|c|}{ Column 1} \\
\hline Total stages & 32 & 26 & 28 \\
\hline Feed stage & 16 & 10 & 11 \\
\hline Reflux Ratio & 13.26 & 14.73 & 18.63 \\
\hline \multicolumn{4}{|c|}{ Column 2} \\
\hline Total stages & 19 & 28 & 24 \\
\hline Feed stage & 7 & 17 & 8 \\
\hline Reflux Ratio & 3.56 & 2.14 & 3.62 \\
\hline \multicolumn{4}{|c|}{ Column 3} \\
\hline Total stages & 13 & 17 & 25 \\
\hline Feed stage & 4 & 9 & 11 \\
\hline Reflux Ratio & 6.16 & 1.70 & 0.90 \\
\hline \multicolumn{4}{|c|}{ Purity } \\
\hline \multirow{4}{*}{ Components } & $\mathrm{C} 1=0.986$ & $\mathrm{C} 1=0.989$ & $\mathrm{C} 1=0.999$ \\
\hline & $\mathrm{C} 2=0.986$ & $\mathrm{C} 2=0.985$ & $\mathrm{C} 2=0.991$ \\
\hline & $\mathrm{C} 3=0.987$ & $\mathrm{C} 3=0.985$ & $\mathrm{C} 3=0.991$ \\
\hline & $\mathrm{C} 4=0.997$ & $\mathrm{C} 4=0.991$ & $\mathrm{C} 4=0.999$ \\
\hline
\end{tabular}

interesting features of the best and worst design generated by BUMDA and NSGA-II (see Table II). It is possible to observe that in energy terms the best solution of the BUMBA algorithm has a heat duty approximately one thousand BTU/h less than the best reported by the NSGA-II. Regarding the number of states, the NSGA-II shows a superior value larger in 6 stages. Likewise, the feed stage location in the column 1 and column 3 are only slightly different on both optimizers, while in the case of column 3 the feed stage corresponds is the 17 in the case of the BUMDA, whereas for the NSGA-II it is located on the stage 8 . Besides, the reflux ratio values determined in column 1 and column 2 for the best in NSGA-II design are higher than the best of the BUMDA. This is due to the combined effect between the stages number and the feed stage location. Nonetheless, the situation is inverted in the column 3 , where the best of the BUMDA works with a superior reflux ratio than the NSGA-II. On the other side, taking into consideration the worst solution found by the BUMDA algorithm it can be noted that in general it has fewer total stages in the column 1, but in the others columns the stages number is less than the best designs presented, with its feed stage located above the feed stage of the best designs, particularly in the column 2 and column 3. However, in the column 1 it was placed below in relation with the best designs. Also, it is important to mention that the reflux ratio values were found similar at the best designs for column 2, however, in the case of columns 1 and 3, this value was significantly superior for the worst design. With all of this, the worst fitness function found was on average $53 \%$ percent greater than the best designs, but its stages number were $12 \%$ less compared with the best of the BUMDA and $21 \%$ less relative to the best of the NSGA-II. Finally, a simple analysis of the target values (purities) of the problem shows that none of the two algorithms did exactly match the target value, however, these targets were better achieved in the case of the BUMDA algorithm (due to the dynamic penalization strategy BUMDA achieved closer results to the target value).

Finally, regarding the performance of the optimization algorithms, it is interesting to highlight that the BUMDA algorithm improves NSGA-II with the proposed penalized
TABLE III. COMPARATIVE ANALYSIS

\begin{tabular}{c||c||c}
\hline \multicolumn{1}{c||}{ Parameter } & \multicolumn{2}{c}{ Case 1: } \\
& Distillation train \\
\cline { 2 - 3 } & BUMDA & NSGA-II \\
\hline \hline Time $(h)$ & 6 & 240 \\
Function evaluation number & 3000 & 100000 \\
Number of individuals & 60 & 2500 \\
Number of generations & 50 & 40 \\
Fitness function $Q, M B T U / h$ & 7.30 & 8.23 \\
\hline
\end{tabular}

fitness function. In Table III, a comparative analysis among them is presented. Note that the computing time required to carry out the optimization by the NSGA-II is 40 times greater than the BUMDA algorithm time. Also, the NSGA-II performs 98\% more function evaluations than the BUMDA algorithm.

This study verifies that the approach presented in this paper, BUMDA with constraint handling, is a powerful and robust tool capable of optimizing processes in chemical engineering, and potentially useful in other engineering areas.

\section{CONCLUSIONS}

A design methodology for generating (near) optimal designs of distillation column sequences that separate mixtures with $N$ components has been presented. A constraint handling technique suitable for distillation columns was attached to the Boltzmann Univariate Marginal Distribution Algorithm (BUMDA). Aspen Plus(C)was used for the rigorous evaluation of the fitness function.

This approach incorporates the design goals as equality constraints through a constraint handling method based on penalty functions which uses the summation of constraint violations. The maximum number of stages is also managed but as an inequality constraint. The most important conclusion is that the best design was found with low numeric effort, short computing time and good success rate, highlighting the fact that the reinitialization mechanism of the variance allowed major exploration on the search space and diversification of the population, reducing the possibility of remaining on local optimal; giving as result best values along of the optimization process. The presented approach improves the NSGA-II results from any perspective.

\section{REFERENCES}

[1] Andrecovich, M.J., Westerberg, A.W., 1985, An MILP Formulation for Heat-Integrated Distillation Sequence Synthesis, AIChE J., 31, 1461.

[2] Paules, G.E., IV, Floudas, C.A., 1990, A Mixed-Integer Nonlinear Programming Formulation for the Synthesis of Heat-Integrated Distillation Sequences, Comput. Chem. Eng., 4, 1397.

[3] Viswanathan, J.; Grossmann, I.E., 1993, Optimal Feed Locations and Number of Trays for Distillation Columns with Multiple Feeds, Ind. Eng. Chem. Res., 32, 2942.

[4] Novak, Z., Kravanja, Z., Grossmann, I.E., 1996, Simultaneous Synthesis of Distillation Sequences in Overall Process Schemes Using an Improved MINLP Approach, Comput. Chem. Eng., 20, 1425.

[5] Smith, E.M.B., Pantelides, C.C., 1995, Design of Reaction/Separation Networks using Detailed Models, Comput. Chem. Eng, 19, S83.

[6] Papalexandri, K.P., Pistikopoulos, E.N., 1996, Generalized Modular Representation Framework for Process Synthesis, AIChE J., 42, 1010.

[7] Bauer, M.H., Stichlmair, J., 1998, Design and Economic Optimization of Azeotropic Distillation Processes Using Mixed-Integer Nonlinear Programming, Comput. Chem. Eng., 22, 1271. 
[8] Dunnebier, G.; Pantelides, C.C., 1999, Optimal Design of Thermally Coupled Distillation Columns, Ind. Eng. Chem. Res., 38, 162.

[9] Caballero, J.A., Grossmann, I.E., 1999, Aggregated Models for Integrated Distillation Systems, Ind. Eng. Chem. Res, 38, 2330.

[10] Yeomans, H., Grossmann, I.E., 2000, Disjunctive Programming Models for the Optimal Design of Distillation Columns and Separation Sequences, Ind. Eng. Chem. Res. 39, 1637.

[11] Martínez-Iranzo, M., Herrero, J.M., Sanchis, J., Blasco, X., GarcíaNieto, S., 2009,Applied Pareto Multi-Objective Optimization by Stochastic Solvers, Engineering Applications of Artificial Intelligence, 22, 455.

[12] Fraga, E.S., Matias T.R., 1996, Synthesis and Optimization of a Nonideal Distillation System Using a Parallel Genetic Algorithm, Comp. Chem. Eng., 20, 79.

[13] Leboreiro, J., Acevedo, J., 2004, Processes Synthesis and Design of Distillation Sequences Using Modular Simulators: A Genetic Algorithm Framework, Comput. Chem. Eng., 28, 1223.

[14] Gómez Castro, F.I., Segovia - Hernández, J.G., Hernández, S., Gutiérrez Antonio, C., Briones - Ramírez, A., 2008, Dividing Wall Distillation Columns: Optimization and Control Properties, Chemical Engineering \& Technology, 31, 1246.

[15] Gutiérrez-Antonio, C., Briones-Ramírez, A., 2009, Pareto Front of Ideal Petlyuk Sequences Using a Multiobjective Genetic Algorithm with Constraints, Comput. Chem. Eng, 33, 454.

[16] Miranda-Galindo, E.Y., Segovia-Hernández, J.G., Hernández, S., Gutiérrez-Antonio, C., Briones-Ramírez, A., 2011, Reactive Thermally Coupled Distillation Sequences: Pareto Front, Ind. Eng. Chem. Res., 50, 926.

[17] Thompson, R.W., and King C.J., 1972, Systematic synthesis of separation systems, AICHE. J., 18, 941.

[18] Caballero, J.A., Grossmann, I.E. Generalized Disjunctive Programming Model for the Optimal Synthesis of Thermally Linked Distillation Columns, Ind. Eng. Chem. Res. 2001, 40, 2260- 2274.

[19] Caballero, J.A., Grossmann, I.E., 2004, Design of Distillation Sequences: from conventional to fully thermally coupled distillation systems. Comput. Chem. Engng., 28, 2307-2329.

[20] Barttfeld, M., P.A. Aguirre and I.E. Grossmann., 2004 A Decomposition Method for Synthesizing Complex Column Configurations Using Tray-by-Tray GDP Models, Computers and Chemical Engineering, 28, 21652188.

[21] Grossmann, I. E., 2002 Review of Non-Linear Mixed Integer and Disjunctive Programming Techniques for Process Systems Engineering, Optimization and Engineering, 2, 227-252.

[22] Grossmann, I. E., 2002 Review of Non-Linear Mixed Integer and Disjunctive Programming Techiques for Process Systems Engineering, Optimization and Engineering, 3, 227-252.

[23] Holland, J. H. 1975/1992, Adaptation in Natural and Articial Systems, Cambridge, MA: MIT Press. Second edition, 1992. First edition, University of Michigan Press, 1975.

[24] Goldberg, D.E., 1982, A Simple Genetic Algorithm: Computer Program in Pascal, Ann Arbor: University of Michigan, Department of Civil Engineering.

[25] Fraga, E.S., Matias T.R., 1996, Synthesis and Optimization of a Nonideal Distillation System Using a Parallel Genetic Algorithm, Comp. Chem. Eng., 20, 79.

[26] Valdez Ivvan, Hernández Arturo, Botello Salvador. A Boltzmann based Estimation of Distribution Algorithm. Information Sciences, ELSEVIER, online version available at: http://www.sciencedirect.com/science/article/pii/S0020025513001576

[27] Kolbe, B., and Wenzel, S., 2004. Nobel distillation concepts using oneshell columns, Chem.Eng. Pross., 43, 339.

[28] Tarafder, A., Rangaiah, G.P., and Ajay K.R., 2007, A Study of Finding Many Desirable Solutions in Multiobjective Optimization of Chemical Processes, Comp. and Chem. Eng., 31, 1257.

[29] Gutiérrez-Antonio, C., 2007, Analisis, Diseño y Optimización de Columnas de Destilación Azeotrópicas, Doctorado en Ciencias en Ingenieria Quimica, Instituto Tecnologico de Celaya, Mexico.

[30] Gómez, C.F.I., Segovia, H. J.G., Hernández, S., Gutiérrez, A.C., and
Briones, R.A., 2008, Dividing wall distillation columns: Optimization and control properties, Chem. Eng. \& Tec., 31, 1246.

[31] Vázquez- Castillo, J.A., Venegas J.A., SegoviaHernández J.G., Hernández-Escoto H., Hernández S., GutiérrezAntonio C., BrionesRamírez A., 2009, Design and optimization, using genetic algorithms, of intensified distillation systems for a class of quaternary mixtures, Comp. and Chem. Eng., 33, 1841.

[32] Gutiérrez-Antonio C., and Briones R.A., 2009, Pareto front of ideal Petlyuk sequences using a multiobjective genetic algorithm with constraints, Comp. and Chem. Eng., 33, 454.

[33] María Vázquez-Ojeda, Juan Gabriel Segovia-Hernández, Salvador Hernández, Arturo Hernndez-Aguirre, Anton Alexandru Kiss. Design and optimization of an ethanol dehydration process using stochastic methods. Separation and Purification Technology, 105, 2013, pp 90-97.

[34] Errico, M., Rong, B.G., Tola, G., Turunen, I., 2009, A Method for Systematic Synthesis of Multicomponent Distillation Systems with Less than N-1 Columns, Chemical Engineering and Processing: Process Intensification, 48, 907.

[35] Errico, M., Tola, G., Mascia, M., 2009, Energy Saving in a Crude Distillation Unit by a Preflash Implementation, Applied Thermal Engineering, 29, 1642.

[36] Petlyuk, F.B., Platonov, V. M., and Slavinskij, D.M., 1965, Thermodynamically Optimal Method for Separating Multicomponent Mixtures, Int. Chem. Eng., 5, 555.

[37] J. Cortez-González, J. G. Segovia-Hernández, S. Hernández, C. Gutiérrez-Antonio, A. Briones-Ramírez, B. G. Rong, 2012, Optimal design of distillation systems with less than $N-1$ columns for a class of four component mixtures, Chemical Engineering Research and Development, 90, 1425-1447.

[38] Ramanathan, S.P., Mukherjee S., Dahule R.K., 2001, Optimization of Continuous Distillation Columns using Stochastic Optimization Approaches, Transactions IChemE, Vol. 79, Part A, Institure of Chemical Engineers.

[39] Bozorgmehry R., Pourahmadi A., Svrcek W., 2009, Automatic Design of Conventional Distillation Column Sequence by Genetic Algorithms, The Canadian Journal of Chemical Engineering, Vol. 87, 477-492, June 2009.

[40] Bozorgmehry R., Pourahmadi A., Svrcek W., 2012, Evolutionary Design of Optimum Distillation Columns Sequence, The Canadian Journal of Chemical Engineering, Vol. 90, 956-972, August 2012 


\section{BUMDA (Boltzmann Univariate Marginal Distribution Algorithm)}

1) Give the parameter and stopping criterion:

nsample $\leftarrow$ Number of individuals to be sample.

minvar $\leftarrow$ minimum variance allowed before restart

2) Uniformly generate the initial population $P_{0}$, set $t=0$.

3) While $t<\operatorname{maxgenerations}$

a) $t \leftarrow t+1$

b) Evaluate fitness $\mathbf{g}(\mathbf{x})$ with constraints as shown in Figure 4, sort, and store the best $20 \%$ of the population

c) Call this set the selected set. The size of this set is nselect.

d) Compute the approximation to $\mu$ and $\sigma^{2}$ (mean and variance for either dimension) with the selected set, as follows:

$\mu \approx \frac{\sum_{1}^{n s e l e c} x_{i} \bar{g}\left(x_{i}\right)}{\sum_{1}^{n s e l e c} \bar{g}\left(x_{i}\right)}, \quad \sigma^{2} \approx \frac{\sum_{1}^{n s e l e c} \bar{g}\left(x_{i}\right)\left(x_{i}-\mu\right)^{2}}{1+\sum_{1}^{n s e l e c} \bar{g}\left(x_{i}\right)}$,

where $\bar{g}\left(x_{i}\right)=g(x)-g\left(x_{n \text { selec }}\right)+1$.

Note: the individuals can be sorted to simplify the computation, and $g\left(x_{n s e l e c}\right)$ is the minimum (for maximization case) objective value of the selected individuals.

e) If $\sigma^{2}<$ minvar then $\left\{\sigma^{2}=\right.$ minvar; minvar=minvar $\left.\times 0.15\right\}$

f) Generate nsample - 1 individuals from a Normal model $N\left(\mu, \sigma^{2}\right)$, and insert the elite individual.

4) Return the elite individual as the best approximation to the optimum.

Fig. 3. Pseudo-code for BUMDA 